\title{
Arginina como aminoácido funcional en dietas para cerdos en iniciación
}

\section{Arginine as functional aminoacido in diets for nursery pigs}

\author{
José Luis Figueroa-Velasco $\left[\right.$ [0, José Alfredo Martínez-Aispuro ${ }^{1 *}\left[\right.$, José Luis Cordero-Mora ${ }^{[}$, María Teresa

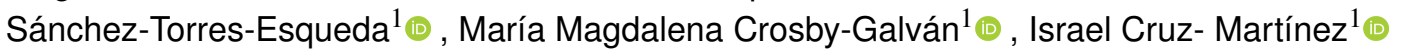 \\ ${ }^{1}$ Programa de Ganadería, Campus Montecillo, Colegio de Postgraduados. Km. 36.5 Carretera México-Texcoco. CP. 56230. Montecillo, \\ Texcoco, Estado de México, México. \\ *Autor de correspondencia: alfredo_aispuro@yahoo.com
}

Nota científica recibida: 09 de febrero de 2018 aceptada: 29 de marzo de 2019

RESUMEN. El objetivo de esta investigación fue determinar la concentración óptima de arginina (Arg) sintética que se debe adicionar a dietas para cerdos en iniciación para mejorar el comportamiento productivo. En el experimento se evaluó la adición de cinco concentraciones de Arg sintética (0.0, 0.5, 1.0, 1.5 y 2.0\%), con 40 cerdos híbridos castrados, con peso inicial promedio de $8.72 \pm 1.36 \mathrm{~kg}$ y 30 días de edad. El diseño utilizado fue completamente al azar con cinco tratamientos. Los datos se analizaron con ANOVA y las medias de tratamientos se compararon con la prueba de Tukey $(\mathrm{p} \leq 0.05)$. La adición a partir de $0.5 \%$ de Arg en la dieta mejoró el consumo de alimento, la ganancia de peso y la conversión alimenticia $(p<0.05)$. La concentración de urea en plasma se incrementó con la adición de $2 \%$ de Arg en la dieta $(p<0.05)$.

Palabras clave: Arginina, aminoácido funcional, características de la canal, cerdos, comportamiento productivo.

ABSTRACT. The objective of this research was to determine the optimum concentration of synthetic arginine (Arg) that should be added to diets of nursery pigs to improve the productive performance. The addition of five concentrations of synthetic $\operatorname{Arg}(0.0,0.5,1.0,1.5$ and 2.0\%) in diets was evaluated with 40 hybrid (Yorkshire $\times$ Duroc $\times$ Pietrain) barrows, with average initial live weight of $8.72 \pm 1.36 \mathrm{~kg}$ and 30 days of age. The design used was completely randomized with five treatments and eight repetitions. The data were analyzed with ANOVA and treatment means were compared with Tukey's test $(p \leq 0.05)$. The addition of $0.5 \%$ Arg in the diet improved average daily feed intake, average daily gain and gain: feed ratio $(p<0.05)$. The plasma urea concentration increased with the addition of $2 \% \operatorname{Arg}$ in the diet $(p<0.05)$.

Key words: Arginine, functional amino acid, carcass characteristics, pigs, productive performance.

\section{INTRODUCCIÓN}

La suplementación con algunos aminoácidos (AA; arginina, glutamina, glutamato, leucina y prolina) en la dieta de humanos y animales modula la expresión de genes, mejora el crecimiento intestinal y el músculo esquelético, y reduce la grasa corporal excesiva (Wu 2014). Estos hallazgos condujeron al nuevo concepto de AA funcionales, que se definen como aquellos que participan y regulan las vías metabólicas claves para mejorar la salud, la supervivencia, el crecimiento, el desarrollo, la lactancia y la reproducción de los organismos (Wu 2013). En la nutrición del cerdo se necesita comprender los roles y requerimientos dietéticos de los $A A$ en cer- dos jóvenes para mejorar la eficiencia de la utilización de proteínas dietéticas y minimizar la excreción de nitrógeno al ambiente (Rezaei et al. 2013); ya que los requerimientos dietéticos de los $A A$ dependen de la especie, la etapa de desarrollo, el estado fisiológico, la microbiota del intestino delgado, factores ambientales y estados patológicos (Dai et al. 2012).

La arginina (Arg) es uno de los AA funcionales más estudiados porque interviene en la respuesta antioxidante, la neurotransmisión, la inmunidad, la síntesis de urea (Wu et al. 2014), el crecimiento de la microbiota intestinal (Dai et al. 2012), la producción de óxido nítrico y la regulación de la expresión génica (Wu et al. 2012); todas ellas actividades básicas para el crecimiento, desarrollo y reproducción animal $\mathrm{Wu}$ 
et al. 2013). Al respecto se sabe que la síntesis endógena de Arg indica un buen comportamiento productivo en cerdos adultos (Ma et al. 2015), por lo cual no se considera como un AA esencial (NRC 2012). Pero cuando el animal se encuentra bajo estrés $y / o$ en periodo de inmunodepresión, los requerimientos de Arg se incrementan (Wijnands et al. 2015), convirtiéndose en un AA semiesencial (NRC 2012).

En lechones, la síntesis endógena de Arg es inadecuada para que manifieste el máximo crecimiento (Kim et al. 2004, Wu et al. 2004), debido a que el cerdo al destete se encuentra en proceso de estrés oxidativo, maduración intestinal y es establecimiento de su sistema inmune. Por lo que la Arg puede ser condicionalmente esencial, por sus mayores tasas de síntesis en esta etapa (Zheng et al. 2013); además, en cerdos al destete, cerca del $40 \%$ de la arginina consumida en la dieta se catabolizada en el intestino delgado (Wu et al. 2004). Por lo anterior, el objetivo del estudio fue determinar la concentración adecuada de Arg sintética que se debe adicionar a dietas de cerdos en etapa de iniciación para mejorar las variables productivas, características de la canal y la concentración de urea en plasma.

\section{MATERIALES Y MÉTODOS}

Los procedimientos experimentales se realizaron cumpliendo con la norma NOM-062-Z001999 para el uso de animales en experimentación (SAGARPA 1999). El estudio se realizó en la Unidad Porcina de la Granja Experimental del Colegio de Postgraduados, localizada a $98^{\circ} 48^{\prime} 27^{\prime \prime}$ LO y a $19^{\circ}$ 48' 23" LN con altitud de $2241 \mathrm{~m}$, en Montecillo, Estado de México. El clima es templado subhúmedo con lluvias en verano, temperatura media anual de $15.2{ }^{\circ} \mathrm{C}$ y precipitación media anual de $644.8 \mathrm{~mm}$ (García 1988).

Las unidades experimentales fueron 40 cerdos (machos castrados) híbridos (Landrace $\times$ Yorkshire $\times$ Duroc), con peso vivo inicial (PVI) promedio de $8.72 \pm 1.36 \mathrm{~kg}$ y 30 días de edad. Los cerdos se alojaron en corrales individuales equipados con comedero tipo tolva y bebedero de chupón. El alimento y el agua se ofrecieron a libre acceso.
Los tratamientos consistieron en la adición creciente de cinco concentraciones de Arginina sintética (L-Arg.HCl, 985\% de pureza; Ajinomoto Inc.): 0.0 , $0.5,1.0,1.5$ y $2.0 \%$. Las dietas para la etapa de iniciación $(8-20 \mathrm{~kg})$ de cada uno de los tratamientos se presentan en la Tabla 1. Los requerimientos nutricionales se establecieron de acuerdo a lo recomendado por el NRC (2012) para cubrirlos o excederlos. Las dietas fueron formuladas con el comando Solver de Microsoft Excel 2016 para cubrir o exceder los requerimientos, bajo el precepto de formulación de dietas al mínimo costo.

\section{Análisis químicos de las dietas}

La proteína cruda se determinó por el método de macrokjeldahl (AOAC 2005). La concentración de calcio y fósforo se determinó por espectrofotometría con un espectrofotómetro de absorción atómica Perkin Elmer 4000, serie Lambda 2, Perkin Elmer (Karl et al. 1979). Al final del experimento se obtuvieron muestras de sangre de la vena cava anterior con tubos Vacutainer ${ }^{\circledR}$ con heparina, que se colocaron en hielo hasta centrifugarse a $2500 \mathrm{~g}$ por 20 min en una centrífuga SIGMA 2-16k, para separar el plasma del paquete celular. El plasma se transfirió a tubos de polipropileno y se almacenó en un congelador SANYO MDF-436 a $-20{ }^{\circ} \mathrm{C}$ hasta la determinación de urea en plasma (Chaney y Marbach 1962).

\section{Variables de respuesta}

Las variables de respuesta fueron: comportamiento productivo (consumo de alimento, CAL; ganancia diaria de peso, GDP; conversión alimenticia, CA; ganancia de carne magra, GCM; y peso vivo final de los cerdos, PVF); características de la canal (grasa dorsal, GD; porcentaje de carne magra, PCM; área del músculo Longissimus dorsi, $\mathrm{AML}$ ); y concentración de urea en plasma (CUP). La GD y AML se midieron utilizando un ultrasonido de tiempo real SonoVet 600 marca Medison al inicio y al final del experimento. Con estos datos y con los de PVI y PVF se calculó la GCM y el PCM (Burson y Berg 2001). 
Figueroa-Velasco et al.

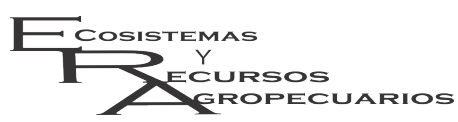

Arginina para cerdos en iniciación

Ecosist. Recur. Agropec.

6(17):377-382,2019

Tabla 1. Composición de las dietas para cerdos en iniciación adicionadas con arginina sintética.

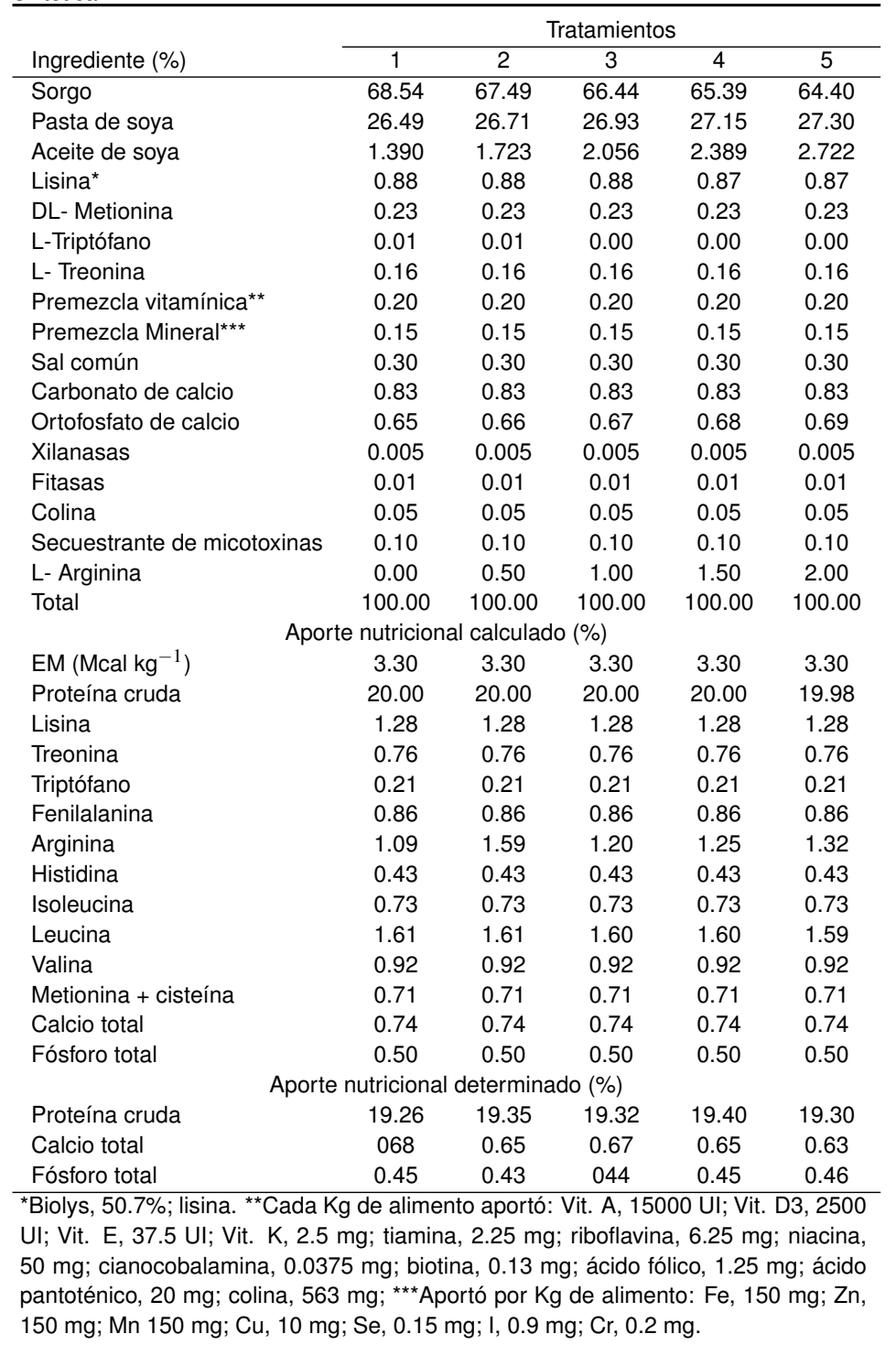

\section{Análisis estadístico}

Los resultados se analizaron bajo un diseño completamente al azar con cinco tratamientos y ocho repeticiones, considerando cada cerdo como unidad experimental. La normalidad y homogeneidad de los datos se evaluaron con las pruebas de Shapiro-Wilk y Levene's. Con los datos se realizó un análisis de varianza mediante el procedimiento GLM y la comparación de medias se realizó con la prueba de Tukey $(p \leq 0.05)$ todos los análisis se realizaron con el programa SAS (SAS 2013). El peso inicial se utilizó como covariable debido a la heterogeneidad de peso observada en los animales $(p \leq 0.05)$.

\section{RESULTADOS Y DISCUSIÓN}

Los resultados para las variables productivas, características de la canal y urea en plasma se presentan en la Tabla 2. La adición de Arg en la dieta incrementó la GDP, PVF y mejoró la CA $(p<0.05)$ con respecto al tratamiento sin Arg, independientemente de la concentración de dicho AA en la dieta. 
Tabla 2. Efecto de la adición de arginina en dietas para cerdos en iniciación.

\begin{tabular}{lccccccccc}
\hline \% Arginina & \multicolumn{4}{c}{ Comportamiento productivo } & \multicolumn{5}{c}{ Características de la canal } \\
& $\begin{array}{c}\mathrm{CDA} \\
(\mathrm{kg})\end{array}$ & $\begin{array}{c}\mathrm{GDP} \\
\left(\mathrm{kg} \mathrm{d}^{-1}\right)\end{array}$ & $\mathrm{CA}$ & $\begin{array}{c}\mathrm{PVf} \\
(\mathrm{kg})\end{array}$ & $\begin{array}{c}\mathrm{GCM} \\
\left(\mathrm{kg} \mathrm{d}^{-1}\right)\end{array}$ & $\begin{array}{c}\mathrm{GD} \\
(\mathrm{mm})\end{array}$ & $\begin{array}{c}\mathrm{AML} \\
\left(\mathrm{cm}^{2}\right)\end{array}$ & $\begin{array}{c}\text { PCM } \\
(\%)\end{array}$ & $\left(\mathrm{mg} \mathrm{dL}^{-1}\right)$ \\
\hline 0.0 & $0.88^{a b}$ & $0.48^{b}$ & $1.85^{a}$ & $18.78^{b}$ & 0.24 & 2.95 & 12.70 & $37.36^{a}$ & $15.17^{b}$ \\
0.5 & $0.93^{a}$ & $0.57^{a}$ & $1.66^{b}$ & $20.61^{a}$ & 0.25 & 3.02 & 12.55 & $35.67^{a b}$ & $18.11^{a b}$ \\
1.0 & $0.88^{a b}$ & $0.60^{a}$ & $1.49^{b}$ & $21.23^{a}$ & 0.25 & 3.11 & 11.74 & $34.15^{b}$ & $18.64^{a b}$ \\
1.5 & $0.87^{a b}$ & $0.58^{a}$ & $1.50^{b}$ & $20.88^{a}$ & 0.26 & 3.02 & 12.78 & $35.82^{a b}$ & $19.11^{a b}$ \\
2.0 & $0.84^{b}$ & $0.54^{a b}$ & $1.57^{b}$ & $20.01^{a b}$ & 0.24 & 2.88 & 12.39 & $35.85^{a b}$ & $20.03^{a}$ \\
EE & 0.048 & 0.02 & 0.046 & 0.36 & 0.01 & 0.15 & 0.59 & 0.70 & 1.15 \\
\hline CDA: consumo diario de alimento, GDP: ganancia diaria de peso, CA: conversión alimenticia, PVf: peso vivo \\
final, GCM: ganancia de carne magra, GD: grasa dorsal, AML: área del músculo Longissimus dorsi, PCM: por- \\
centaje de carne magra, NUP: N ureico en plasma. ${ }^{a, b}$ Medias de tratamiento con distinta literal indica diferencias \\
estadísticas (p $\leq$ 0.05), EE = Error estándar.
\end{tabular}

EI CDA fue mayor $(\mathrm{p}<0.05)$ al comparar la adición de 0.5 y $2.0 \%$ de Arg. En cuanto a las características de la canal, la GD y el AML no fueron afectadas por el consumo de Arg sintética en la dieta; mientras que el PCM disminuyó $(p<0.05)$ al adicionar $1.0 \%$ de Arg con respecto al tratamiento testigo. Al utilizar una concentración mayor de $0.5 \%$ de Arg se observó que la GDP, PVF y la conversión alimenticia no mejoraron; con lo cual se puede inferir que no existe un beneficio adicional al suplementar más de $0.5 \%$ de Arg en la dieta de cerdos en iniciación en las variables productivas y características de la canal. La adición de $2 \%$ de Arg en la dieta modificó la concentración de urea en plasma $(p<0.05)$ observándose que a medida que se incrementa la concentración de Arg dicho metabolito aumentó.

Los mejores valores para CDA, GDP, PVf y CA se presentaron en los animales cuyas dietas fueron suplementadas con $0.5 \%$ de Arg, similar a lo observado en trabajos con lechones suplementados con 0.2 a $0.6 \%$ de Arg, lo que incrementó la GDP y PVf, mejoró la CA y aumentó la síntesis de proteína en músculo Longissimus dorsi; pero no hubo efecto en el CDA (Kim et al. 2004, Wu et al. 2004, Yao et al. 2008). De igual manera en cerdos castrados en etapa de crecimiento la adición de $1 \%$ de Arg muestra efectos favorables en la GDP, características de la canal y síntesis de proteína (He et al. 2009, Tan et al. 2009). Dichos reportes confirman lo obtenido en el presente trabajo, ya que los resultados indican que la concentración más adecuada de Arg sintética en la dieta para cerdos en etapa de lactancia e iniciación fue $0.5 \%$. Esto debido a que una dieta típica a base de sorgo o maíz- pasta de soya, no provee la cantidad suficiente de arginina para la síntesis de proteína en cerdos al destete (Wu et al. 2004).

La Arg es un promotor de crecimiento muscular (McKnight et al. 2010), que se asocia con la activación de reguladores positivos de la síntesis de proteínas y la síntesis fraccional de proteína en el músculo (Yao et al. 2008); lo que permite mayor deposición de proteína en el músculo esquelético de cerdos (He et al. 2009; Tan et al. 2009). En un estudio realizado por $\mathrm{Hu}$ et al. (2015) con cerdos al destete, comprobaron la inocuidad de suplementar hasta $2 \%$ de la ración con L- arginina, encontraron que la adición de L-arginina aumenta la ganancia de proteína corporal. Lo que se ha observado en cerdas suplementadas con Arg desde etapas previas al nacimiento, las cuales presentaron mejores camadas y mejor comportamiento productivo de los lechones durante la lactancia (Mateo et al. 2008, Gao et al. 2012, Kalbe et al. 2013).

Aunque la Arg ha sido asociada como un ingrediente eficaz para disminuir la grasa corporal ( $\mathrm{He}$ et al. 2009, Tan et al. 2009, Wu et al. 2012). Al respecto en la presente investigación el uso de Arg no tuvo efecto en dicha variable. El incremento de la concentración de urea en plasma en respuesta a la adición de Arg en la dieta, puede deberse a que una concentración mayor de $0.5 \%$ Arg en la dieta causa un exceso de este aminoácido en el torrente circulatorio, el cual tiene que ser eliminado, dado que la Arg participa en el ciclo de la urea y es eliminado excretando nitrógeno para mantener la homeostasis en el animal; ya que el aumento de PC total de la dieta in- 
crementa la cantidad de urea en el plasma (González et al. 2014, 2016).

La adición de arginina sintética en dietas para cerdos en etapa de iniciación mejora las variables productivas, pero una adición mayor del $0.5 \%$ no tiene beneficio adicional en el comportamiento productivo.

\section{LITERATURA CITADA}

AOAC (2005) Official Methods of Analysis. Association of Analytical Chemists $18^{\text {th }}$ Ed. Washington, DC, USA. $128 p$.

Burson D, Berg E (2001) Procedures for estimating pork carcass composition. Pork quality facts. National Pork Producers Council, Des Moines IA, USA. https://articles.extension.org/pages/27311/procedures-forestimating-pork-carcass-composition. Fecha de consulta: 25 de enero de 2018.

Chaney AL, Marbach EP (1962) Modified reagents for determination of urea and ammonia. Clinical Chemistry 8 : 130-132.

Dai ZL, Li XL, Xi PB, Zhang J, Wu G, Zhu WY (2012) Regulatory role for L-arginine in the utilization of amino acids by pig small-intestinal bacteria. Amino Acids 43: 233-244.

Gao K, Jiang Z, Lin Y, Zheng C, Zhou G, Chen F, Yang L, Wu G (2012) Dietary L-arginine supplementation enhances placental growth and reproductive performance in sows. Amino Acids 42: 2207-2214.

García E (1988) Modificaciones al sistema de clasificación de Köppen (para adaptarlas a las condiciones de la República Mexicana). $4^{a}$ edición. México. 217p.

González M, Figueroa JL, Vaquera H, Sánchez-Torres MT, Ortega M, Cordero JL, et al. (2014) Niveles de proteína para cerdos en fase starter: un meta-análisis. Archivos de Zootecnia 63: 315-325.

González M, Figueroa JL, Vaquera H, Sánchez-Torres MT, Ortega ME, Copado JMF, et al. (2016) Metaanálisis del efecto de dietas bajas en proteína y adicionada con aminoácidos sintéticos para cerdos machos castrados en finalización. Archivos de Medicina Veterinaria 48: 51-59.

He Q, Kong X, Wu G, Ren P, Tang H, Hao F, et al. (2009) Metabolomic analysis of the response of growing pigs to dietary L-arginine supplementation. Amino Acids 37: 199-208.

Hu S, Li X, Rezaei R, Meininger CJ, McNeal CJ, Wu G (2015) Safety of long-term dietary supplementation with I-arginine in pigs. Amino Acids 47: 925-936.

Kalbe C, Bérard J, Porm M, Rehfeldt C, Bee G (2013) Maternal L-arginine supplementation during early gestation affects foetal skeletal myogenesis in pigs. Livestock Science 157: 322-329.

Karl RF, McDowell LR, Miles PH, Wilkinson NS, Funk JD, Corad JH (1979) Métodos de análisis de minerals para tejidos de plantas y animales. 2a Ed. Departamento de Ciencia Animal, Universidad de Florida. Gainesville. Florida, USA. 39p.

Kim SW, McPherson RL, Wu G (2004) Dietary arginine supplementation enhances the growth of milk-fed young pigs. Journal of Nutrition 134: 625-630.

Ma X, Zheng C, Hu Y, Wang L, Yang X, Jiang Z (2015) Dietary L-arginine supplementation affects the skeletal longissimus muscle proteome in finishing pigs. Plos One 10(1): e0117294 Doi: 10.1371/journal.pone.01172 94.

Mateo RD, Wu G, Moon HK, Carroll JA, Kim SW (2008) Effects of dietary arginine supplementation during gestation and lactation on the performance of lactating primiparous sows and nursing piglets. Journal of Animal Science 86: 827-835. 
McKnight JR, Satterfield MC, Jobgen WS, Smith SB, Spencer TE, Meininger CJ, et al. (2010) Beneficial effects of L-arginine on reducing obesity: potential mechanisms and important implications for human health. Amino Acids 39: 349-57.

NRC (2012) Nutrient requirements tables and feed ingredient composition. Nutrient requirements of swine. National Research Council. $11^{\text {th }}$ Ed. National Academy Press. Washington. 400p.

Rezaei R, Wang WW, Wu ZL, Dai Z, Wang J Wu, G (2013) Biochemical and physiological bases for utilization of dietary amino acids by young pigs. Journal of Animal Science and Biotechnology 4(1): 7 Doi: 10.1186/20491891-4-7.

SAGARPA (1999) Norma Oficial Mexicana-NOM-062-ZOO-1999: Especificaciones técnicas para la producción, cuidado y uso de los animales de laboratorio. Diario Oficial de la Federación. México. http://www.fmvz. unam.mx/fmvz/principal/archivos/062ZOO.PDF. Fecha de consulta: 25 de enero de 2018.

SAS (2013) Base SAS ${ }^{\circledR}$ 9.4. Procedures Guide Statistical Procedures. Second Edition. SAS Institute Inc., Cary, NC, USA. 550p.

Tan B, Yin Y, Liu Z, Li X, Xu H, Kong X, et al. (2009) Dietary L-arginine supplementation increases muscle gain and reduces body fat mass in growing-finishing pigs. Amino Acids 37: 169-175.

Wijnands KAP, Castermans TMR, Hommen MPJ, Meesters DM, Poeze M (2015) Arginine and citrulline and the immune response in sepsis. Nutrients 7: 1426-1463.

Wu G, Knabe DA, Kim SW (2004) Arginine nutrition in neonatal pigs. In: Arginine Metabolism: enzymology, nutrition, and clinical significance. Journal of Nutrition 134: 2783-2790.

Wu Z, Satterfield MC, Bazer FW, Wu G (2012) Regulation of brown adipose tissue development and white fat reduction by L-arginine. Current Opinion in Clinical Nutrition and Metabolic Care 6: 529-538.

Wu G (2013) Functional amino acids in nutrition and health. Amino Acids 45: 407-411.

Wu G, Wu Z, Dai Z, Yang Y, Wang W, Liu C, et al. (2013) Dietary requirements of "nutritionally non-essential amino acids"by animals and humans. Amino Acids 44: 1107-1113.

Wu G (2014) Dietary requirements of synthesizable amino acids by animals: a paradigm shift in protein nutrition. Journal of Animal Science and Biotechnology 5: 34 Doi : 10.1186/2049-1891-5-34.

Wu G, Bazer FW, Dai Z, Li D, Wang J, Wu Z (2014) Amino Acid Nutrition in Animals: Protein Synthesis and Beyond. Annual Review of Animal Biosciences 2: 387-417.

Yao K, Yin YL, Chu W, Liu Z, Deng D, Li T, et al. (2008) Dietary arginine supplementation increases mTOR signaling activity in skeletal muscle of neonatal pigs. Journal of Nutrition 138: 867-872.

Zheng P, Yu B, He J, Tian G, Luo Y, Mao X, Zhang K, Che K, Chen D (2013) Protective effects of dietary arginine supplementation against oxidative stress in weaned piglets. British Journal of Nutrition 109: 2253-2260. 